\title{
Mean-Field Study of the Degenerate Blume-Emery-Griffiths Model in a Random Crystal Field ${ }^{1}$
}

\author{
N. S. Branco ${ }^{a, 2}$ and Luciano Bachmann ${ }^{b}$ \\ ${ }^{a}$ Universidade Federal de Santa Catarina, Depto. de Física \\ 88040-900, Florianópolis, SC, Brazil, e-mail: nsbranco@fisica.ufsc.br \\ ${ }^{\mathrm{b}}$ CNEN - IPEN - Caixa Postal 11049, 05422-970, São Paulo, SP, Brazil
}

\begin{abstract}
The degenerate Blume-Emery-Griffiths (DBEG) model has recently been introduced in the study of martensitic transformation problems. This model has the same Hamiltonian as the standard Blume-Emery-Griffiths (BEG) model but, to take into account vibrational effects on the martensitic transition, it is assumed that the states $S=0$ have a degeneracy $p(p=1$ corresponds to the usual BEG model). In some materials, the transition would be better described by a disordered DBEG model; further, the inclusion of disorder in the DBEG model may be relevant in the study of shape memory alloys. From the theoretical point of view, it would be interesting to study the consequence of conflicting effects: the parameter $p$, which increases the first-order phase-transition region, and disorder in the crystal field, which tends to diminish this region in three dimensions. In order to study this competition in high-dimensional systems, we apply a mean-field approximation: it is then possible to determine the critical behavior of the random DBEG model for any value of the interaction parameters. Finally, we comment on (preliminary) results obtained for a two-dimensional system, where the randomness in the crystal-field has a more drastic effect, when compared to the three-dimensional model.
\end{abstract}

Key words: Multicritical phase-diagrams, disordered models, mean-field approximation.

PACS number(s): 75.10.Hk, 64.60.Ak, 64.60.Kw

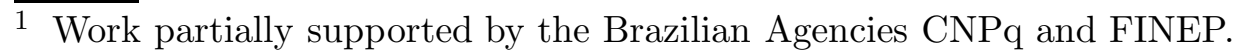

2 Author to whom the proofs should be sent. 


\section{Introduction}

The hamiltonian of the degenerate Blume-Emery-Griffiths (DBEG) model reads: $\mathcal{H}=-J \sum_{<i, j>} S_{i} S_{j}-K \sum_{<i, j>} S_{i}^{2} S_{j}^{2}+\Delta \sum_{i} S_{i}^{2}$, where $J$ is the exchange constant, $K$ is the biquadratic interaction, $\Delta$ is a crystal field, the first two sums are over nearest-neighbor pairs on a lattice, the third sum is over all sites, $S_{i}= \pm 1,0$, and the $S=0$ states have a degeneracy $p$ [1]. This parameter mimics the effects of vibrational degrees of freedom on martensitic transitions. The DBEG model was studied, for a particular value of the crystal-field, within a mean-field approximation and Monte Carlo simulation: the effect of $p$ is to shrink the ferromagnetic phase and to increase the region where the transition is of first-order [1].

Nevertheless, some materials were found to be better described by a disordered DBEG model, which may be relevant in the description of conventional shape memory alloys as well [1]. From the theoretical point of view, on the other hand, it has been shown that randomness may have drastic consequences on multicritical behavior. In two dimensions, for instance, any infinitesimal amount of disorder supresses non-symmetry-breaking first-order phase transitions and replaces symmetry-breaking first-order phase transitions by continuous ones. The effect of disorder on high-dimensional systems is different: first-order phase transitions disappear only at a finite amount of randomness [2]. This behavior has been observed in a large variety of models, either with random "field" or with random "interaction" [2-4].

Thus, in the disordered DBEG model two ingredients have opposite effects: while the degeneracy factor $p$ increases the region where the transition is of first-order, disorder tends to supress this region. This competition may have interesting effects on the phase diagrams, apart from potential experimental relevance. In order to study this competition on high-dimensional systems, we apply a mean-field approximation to the DBEG model in a random crystal- 
field, which follows the probability distribution $\mathcal{P}\left(\Delta_{i}\right)=r \delta\left(\Delta_{i}+\Delta\right)+(1-$ r) $\delta\left(\Delta_{i}-\Delta\right)$. It is worthy stressing that, if the interaction parameters, $J$ and $K$, were chosen to be random, instead of the crystal field, the overall consequences on the phase diagram would be the same [2].

\section{Formalism and Results}

Our approximation is based on the Gibbs inequality for the free energy [5]: $F \leq \operatorname{Tr} \rho \mathcal{H}+(1 / \beta) \operatorname{Tr} \rho \ln \rho$, where $F$ is the exact free energy, $\mathcal{H}$ is the DBEG hamiltonian and $\rho$ is an exactly solvable density matrix. We chose $\rho$ as the most general single-site density matrix for the ferromagnetic (positive $J$ and $K)$ system. Since this procedure is usual, we will not discuss it in detail.

Let us first comment on some general results we obtain, for any $K / J$. The transition at zero temperature is not affected by the parameter $p$ and takes place at $(\Delta / z J)=(K / J+1)(1+r) / 2$; for smaller values of $\Delta / z J$ the magnetization equals 1 , while $m=r$ for $\Delta / z J>(K / J+1)(1+r) / 2$.

For $\Delta / z J=\infty$, the random field DBEG model, with the field distribution given by the equation in the previous page, is equivalent to the quenched sitediluted spin-1/2 Ising model. The states $S= \pm 1$ represent magnetic sites and the states $S=0$ represent non-magnetic impurities. Therefore, only for $r \geq r_{c}$ (where $r_{c}$ is the lattice-dependent value of $r$ where an infinite cluster of present sites is formed for the first time, when $r$ is increased from zero) the critical line between $O 2$ and $D$ (see figures) should extend to $\Delta / J=\infty$. For $r<r_{c}$, the "tail" of continuous transition would touch the zero temperature axis at a finite value of $\Delta / z J$. The reason we find this "tail" extending to $\Delta / J=\infty$ for any $r \neq 0$ is that the present approximation is equivalent to a model with infinite-range interactions (see Ref. [3]).

The assymptotic value of $k T / z J$ for $\Delta / z J=\infty$ equals $r$. This result comes 
from the mapping cited in the last paragraph: the degeneracy of the $S=0$ states and the biquadratic interaction $K$ play no role in the dynamics of the $S= \pm 1$ states. If the distribution $\mathcal{P}\left(\Delta_{i}\right)=r \delta\left(\Delta_{i}\right)+(1-r) \delta\left(\Delta_{i}-\Delta\right)$ is used, a different result will hold. The limit $\Delta / z J=\infty$ is now equivalent to the spin-1 site-diluted Ising model; therefore, $K$ and $p$ are relevant and the assymptotic value of $k T / z J$ depends on both parameters.

Since for high values of $K / J$ the critical behavior does not depend strongly on $p$, we well depict only the phase diagrams for $K / J=0$, where qualitative changes occur when $p$ is varied.

In Figs. 1 and 2 we show the $k T / z J \times \Delta / z J$ phase diagrams for $K / J=0$ and for some values of $p$. For $r=0.1$, the reentrant behavior observed for $p=1$ is lost and a tricritical point is introduced for $p=3$. This latter curve is qualitatively equivalent to the phase diagram for $r$ close to zero. For $r=0.3$ a reentrant behavior is obtained for high enough $p$ (curve(c)); a critical end point (E), which was not present for $p=1$ and $p=3$, is also observed. The first order transition curve for $p=7$ extends to negative values of $\Delta / z J$, until it reaches a line of continuous transitions at a tricritical point (not depicted in the Fig. 2).

\section{Conclusion}

Using a mean-field approximation, we calculate the phase diagrams for the random crystal field DBEG model, varying the degree of disorder, $r$, and the degeneracy factor $p$. For high values of $K / J$ the phase diagrams are almost insensitive to the value of $p$, while for $K / J=0$ the dependence on $p$ is stronger. For the latter value of $K / J$ and $r=0.3$ the phase diagram changes qualitatively when $p$ is increased enough: while for $p=1$, for instance, the order-disorder transition is always continuous and the first-order transition inside the ordered phase ends in a critical point, for $p=7$ a line of first or- 
der transition separates the ordered and disordered phases at low values of $\Delta / z J$. A reentrant behavior is obtained for high $p$. For $K=0$ and $r=0.1$, the degeneracy reintroduces a tricritical point and eliminates the reentrant behavior.

Some of the features found in this work may be artifact of the simple meanfield approximation we used and should not hold for a low-dimensional system. Preliminary results for the square lattice, using a real-space renormalizationgroup approach, show a different behavior: for any infinitesimal amount of disorder the first order transition is supressed, independent of $p$.

\section{References}

[1] E. Vives, T. Castán e P.-A. Lindgård, Phys. Rev. B 53 (1996) 8915.

[2] See, for instance, K. Hui and A. N. Berker, Phys. Rev. Lett. 62 (1989) 2507 or A. N. Berker, J. Appl. Phys. 70 (1991) 5941 and references therein.

[3] N. S. Branco and Beatriz Boechat, to be published in Phys. Rev. B , November 1997.

[4] J. Cardy and J. L. Jacobsen, preprint (cond-mat 9705038).

[5] H. Falk, Am. J. Phys. 38 (1970) 858.

[6] N. S. Branco and Luciano Bachmann, in preparation. 


\section{FIGURE CAPTIONS}

Figure 1: Phase diagram of the random-field DBEG model, for $K / J=0$ and $r=0.1$. Dashed (continuous) lines indicate first-order (continuous) transition, $k$ is the Boltzman constant, $T$ is the temperature and $z$ is the coordination numer of the lattice. $O 1(O 2)$ stands for the ordered phase with $m=1(m=r)$ at $T=0, D$ stands for the disordered phase, $C$ stands for critical points, $E$ stands for critical end points, and $T C$ stands for tricritical points. Finally, (a) stands for $p=1$, while (b) stands for $p=3$. The diagram for $r=0$ is qualitatively the same as the curve (b), except for the $O 2$ phase, which is no longer present.

Figure 2: Phase diagram of the random-field DBEG model, for $K / J=0$ and $r=0.3$. Same notation as in previous figure; $(c)$ stands for $p=7$. Note the qualitative change for $p>3$ : a critical end point is present for $p=7$, as well as reentrant behavior, with order-disorder-order transitions as $\Delta / z J$ is increased from small values. 